\title{
A Global Repair Operator for Capacitated Arc Routing Problem
}

\author{
Yi Mei, Ke Tang, Member, IEEE, and Xin Yao, Fellow, IEEE
}

\begin{abstract}
Capacitated arc routing problem (CARP) has attracted much attention during the last few years due to its wide applications in real life. Since CARP is NP-hard and exact methods are only applicable for small instances, heuristics and metaheuristic methods are widely adopted when solving CARP. This paper demonstrates one major disadvantage encountered by traditional search algorithms and proposes a novel operator named global repair operator (GRO) to address it. We further embed GRO in a recently proposed tabu search algorithm (TSA) and apply the resultant repair-based tabu search (RTS) algorithm to five well-known benchmark test sets. Empirical results suggest that RTS not only outperforms TSA in terms of quality of solutions but also converges to the solutions faster. Moreover, RTS is also competitive with a number of state-of-the-art approaches for CARP. The efficacy of GRO is thereby justified. More importantly, since GRO is not specifically designed for the referred TSA, it might be a potential tool for improving any existing method that adopts the same solution representation.
\end{abstract}

Index Terms-Capacitated arc routing problem (CARP), global repair operator (GRO), heuristic search, tabu search.

\section{INTRODUCTION}

$\mathbf{T}$ HE CAPACITATED arc routing problem (CARP) is a classic problem with wide applications in real world, such as urban waste collection, post delivery, salting route optimization, winter gritting, etc. [1]. It involves determining a minimum cost routing plan for a set of vehicles, each of which is associated with a capacity constraint. Concretely, the CARP can be represented by a graph $G=(V, E, A)$, where the vertex set $V$, the edge set $E$, and the arc set $A$ represent the set of intersections, two-way streets, and one-way streets, respectively. A vertex $\operatorname{dep} \in V$ represents a central depot where a set of vehicles are based. Two subsets $E_{R} \subseteq E$ and $A_{R} \subseteq A$ are called task sets, consisting of tasks required to be served by the vehicles (e.g., the streets required to be cleaned in winter gritting problem). Each element $(t, h) \in E \cup A$ is as-

Manuscript received April 21, 2008; revised August 5, 2008. This work was supported in part by the Fund for Foreign Scholars in University Research and Teaching Programs under Grant B07033 and in part by an Engineering and Physical Science Research Council (EPSRC) grant in U.K. under Grant EP/E058884/1. This paper was recommended by Associate Editor H. Takagi.

Y. Mei and K. Tang are with the Nature Inspired Computation and Applications Laboratory, Department of Computer Science and Technology, University of Science and Technology of China, Hefei 230027, China (e-mail: meiyi@mail.ustc.edu.cn; ketang@ustc.edu.cn).

$\mathrm{X}$. Yao is with the Nature Inspired Computation and Applications Laboratory, Department of Computer Science and Technology, University of Science and Technology of China, Hefei 230027, China, and also with the Centre of Excellence for Research in Computational Intelligence and Applications, School of Computer Science, University of Birmingham, B15 2TT Birmingham, U.K. (e-mail: x.yao@cs.bham.ac.uk).

Digital Object Identifier 10.1109/TSMCB.2008.2008906 sociated with three costs, i.e., $d(t, h), s c(t, h)$, and $d c(t, h)$, which indicates the demand, the cost of serving, and passing without serving from the tail vertex $t$ to the head vertex $h$ (i.e., deadheading), respectively. Note that, for nonrequired edges and arcs, $d(t, h)=0$. The aim is to schedule the routes for each vehicle, so that the total cost of the routes is minimized. Each route starts and ends at the depot, and the total demand processed must not exceed vehicle capacity.

Since CARP is NP-hard [2], exact algorithms are only applicable for small instances. For this reason, various heuristics and metaheuristics have been proposed for CARP. To name a few, Golden and Wong proposed a constructive heuristic called augment-merge [2] in 1981. After that, Golden et al. proposed another constructive heuristic called path scanning [3]. Ulusoy improved path scanning to form a new heuristic called Ulusoy's heuristic [4]. Pearn proposed an approximate algorithm [5] and an augment-insert heuristic [6] for CARP. Mourao and Amado proposed a heuristic method for mixed CARP and demonstrated that it outperforms all the previous heuristics [7]. Amponsah and Salhi proposed an efficient constructive heuristic embedded with a look-ahead strategy and enhancement procedures [8]. Hertz et al. proposed a tabu search for CARP called CARPET [9] and a variable neighborhood descent (VND) algorithm [10]. Greistorfer proposed a tabu scatter search for arc routing problem [11]. Lacomme et al. proposed a competitive memetic algorithm (MA) for CARP [12] and a genetic algorithm for CARP and its extension [13]. Beullens et al. proposed a guided local search (GLS) method [14]. Handa et al. proposed an evolutionary algorithm for the salting route optimization, which is an application of CARP [15], [16]. Recently, Brandão and Eglese proposed a deterministic tabu search algorithm (TSA) [17].

The solution representation of a heuristic method is critical for conducting an effective search and thus has much influence on obtaining good solutions. In the literature of CARP, a solution is commonly encoded as a set of routes, each of which is an ordered list of vertices. Every vertex is associated with a zero-one variable, indicating whether the edge between this vertex and the successive vertex is served. TSA and CARPET directly use such vertex encoding. MA represents solutions as ordered lists of tasks. Since each task corresponds to a unique pair of vertices, MA can be viewed as using the vertex encoding as well.

In the literature, the vertex encoding has provided generally satisfactory results. However, as will be explained in detail in Section II-B, the existing algorithms adopting the vertex encoding are likely to overlook those promising infeasible solutions. Under this circumstance, the search will be ineffective. This paper presents a novel repair operator, namely, the global 


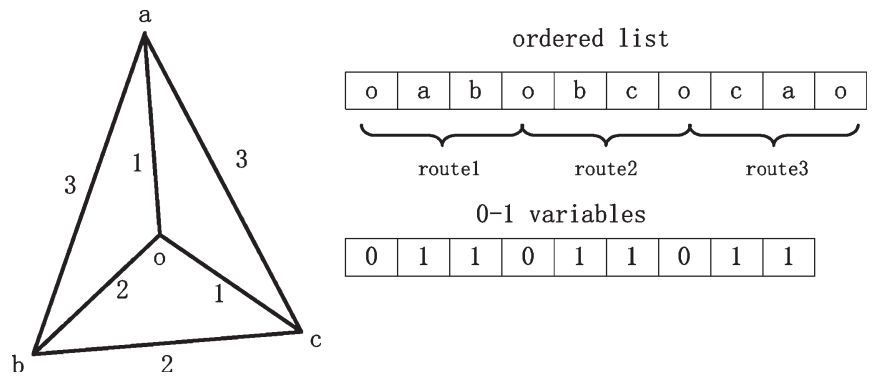

Fig. 1. Vertex encoding for a CARP solution.

repair operator (GRO), to handle such problems. The GRO can be easily embedded in many existing algorithms and improve them significantly in terms of the total cost of the achieved solution. Moreover, GRO requires little computational cost while can accelerate convergence of the original algorithm and, thereby, even shortens the time required to obtain high quality solutions. The advantages of GRO are justified by embedding it in the TSA and comparing with six existing algorithms, i.e., the CARPET, VND, GLS, MA, and two versions of TSA, on five benchmark test sets.

The rest of this paper is organized as follows. First, the mathematical formulation of CARP is presented in Section II, followed by the introduction of GRO. Section III presents the repair-based tabu search (RTS) algorithm, which is obtained by embedding GRO in TSA. Section IV is dedicated to the empirical studies. Finally, the conclusion and discussion are presented in Section V.

\section{REPAIR OPERATOR FOR CARP}

This section describes the proposed GRO. We start from describing the mathematical formulation of CARP. After that, motivation and detailed steps of GRO are presented.

\section{A. Mathematical Formulation of CARP}

Suppose a CARP represented by a graph $G=(V, E, A)$ is given. Let $V=\left\{v_{1}, \ldots, v_{n}\right\}$ and $v_{1}$ denotes the depot; each $\left(v_{i}, v_{j}\right) \in E \cup A$ is associated with the demand $d\left(v_{i}, v_{j}\right)$, serving cost $s c\left(v_{i}, v_{j}\right)$, and deadheading cost $d c\left(v_{i}, v_{j}\right)$. Furthermore, a set of vehicles with identical capacity $Q$ is based at the depot.

As mentioned before, the vertex encoding, which is widely used in heuristic approaches to CARP, consists of an ordered list and a vector of zero-one variables. The ordered list is a permutation of vertices. More specifically, an ordered list consists of several routes, each of which starts and ends with $v_{1}$. Every element of the ordered list is associated with a zero-one variable $y . y$ takes one if the edge between the vertex and its successor is served at this stage of the route. Fig. 1 shows the vertex encoding for a solution of a CARP. $a, b, c$, and $o$ are vertices, where $o$ denotes the depot. The first permutation is the ordered list, which can be divided into three single routes named route1, route2, and route3, respectively. Each route starts and ends at the depot $o$, and traverses the vertices in the order given by the ordered list, e.g., route1 leaves $o$, visits $a$ and $b$ consequently, and then returns $o$. The second vector is the associated zero-one variable vector. The solution corresponding to such a vertex encoding is as follows: Route1 traverses $(o, a)$ deadheadingly and then serves $(a, b)$ and $(b, o)$; route 2 traverses $(o, b)$ deadheadingly and then serves $(b, c)$ and $(c, o)$; route 3 traverses $(o, c)$ deadheadingly and then serves $(c, a)$ and $(a, o)$.

An ordered list can be formally presented as $O L=$ $\left\langle l_{1}, l_{2}, \ldots, l_{K}\right\rangle$, where $l_{k} \in V$, with $k=1,2, \ldots, K$. Suppose there are $m$ routes in the ordered list, then the depot $v_{1}$ appears $(m+1)$ times in the ordered list. Without loss of generality, assume these $v_{1}$ 's lie at the position $r_{0}, r_{1}, \ldots, r_{m}$, where $r_{0}=1, r_{m}=K$, and $r_{i}<r_{j}, \forall 1 \leqslant i<j \leqslant m$. Accordingly, the zero-one variable vector is denoted as $\left\langle y_{1}, y_{2}, \ldots, y_{K-1}\right\rangle$. $y_{k}$ takes one if $\left(l_{k}, l_{k+1}\right)$ is served at position $k$ of the ordered list and takes zero if otherwise.

With the aforementioned definitions, the CARP can be formulated as follows:

$$
\begin{aligned}
& \min \sum_{k=1}^{K-1}\left(s c\left(l_{k}, l_{k+1}\right) \times y_{k}+d c\left(l_{k}, l_{k+1}\right) \times\left(1-y_{k}\right)\right) \\
& \text { s.t. : } \sum_{k=1}^{K-1} y_{k}=N \\
& \left(l_{k}, l_{k+1}\right) \in E_{R} \cup A_{R} \quad \forall y_{k}=1 \\
& \left(l_{k}, l_{k+1}\right) \neq\left(l_{j}, l_{j+1}\right) \quad \forall y_{k}=1 ; \quad y_{j}=1 \\
& k \neq j \\
& \sum_{k=r_{i}}^{r_{i+1}-1} d\left(l_{k}, l_{k+1}\right) \times y_{k} \leqslant Q, \quad i=0,1,2, \ldots, m-1 \\
& l_{k} \in V, \quad k=1,2, \ldots, K \\
& \left(l_{k}, l_{k+1}\right) \in E \cup A, \quad k=1,2, \ldots, K-1 \\
& y_{k}=0 \text { or } 1, \quad k=1,2, \ldots, K-1
\end{aligned}
$$

where $N$ is the number of tasks. Constraints (2)-(4) indicate that each task is served once and only once. Constraint (5) bounds the total demand of each single route with the vehicle capacity. Constraints (6)-(8) define the domains of the variables.

\section{B. Motivation}

Substituting constraints (2)-(4) into the objective function (1) in the former section, we can easily modify function (1) to the following form:

$$
\min \sum_{k=1}^{K-1}\left(d c\left(l_{k}, l_{k+1}\right)\right)+\sum_{(i, j) \in E_{R} \cup A_{R}}(s c(i, j)-d c(i, j)) .
$$

From the modified objective function, it can be observed that the two components of vertex encoding play different roles in the solution. The ordered list determines the total cost of a solution, while the zero-one variables determine whether and how much the solution violates the capacity constraint. Since an edge can be traversed multiple times in CARP, one ordered 


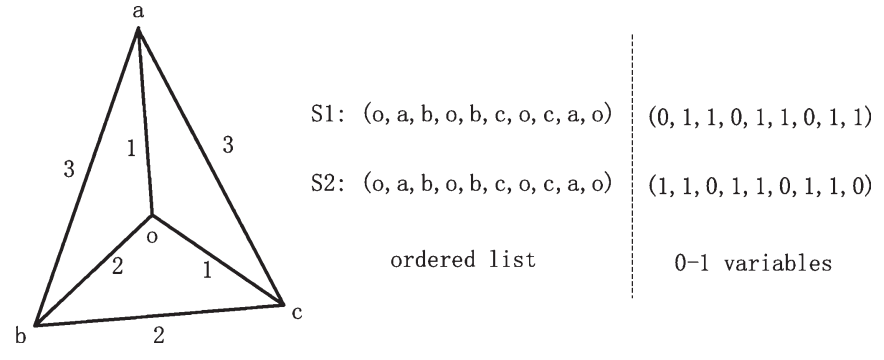

Fig. 2. Example of CARP.

list may correspond to many solutions with different values of the zero-one variables.

The optimal solution of CARP corresponds to both an optimal ordered list and a feasible assignment of zero-one variables on the ordered list. In the existing methods, various search operators have been employed, such as single insertion (SI), double insertion (DI), swap (employed in MA [12] and TSA [17]), and 2-opt (employed in MA [12]). Given the current solution, all these operators generate a new solution by modifying both the ordered list and the corresponding zero-one variables. However, the simultaneous optimization of the two components is not an easy task, and modifying them simultaneously may even lead to an ineffective search in the solution space. An example is given in Fig. 2 to demonstrate this problem. In Fig. 2, there are four vertices in the graph, denoted as $a, b, c$, and $o$, of which vertex $o$ represents the depot. All the six edges, i.e., $\{(o, a),(o, b),(o, c),(a, b),(a, c),(b, c)\}$, are required to be served. Suppose that there are three vehicles available, with the same capacity of four. The number on each edge denotes its demand, e.g., $d(a, b)=3$. The right part in Fig. 2 shows the vertex representations of two different solutions $S_{1}$ and $S_{2} . S_{1}$ and $S_{2}$ share the same ordered list but are different on the second component. As a result, $S_{1}$ is infeasible with one unit capacity violation, i.e., $d(a, b)+d(b, o)=3+2=5>4$, whereas $S_{2}$ is the global optimum. Hence, given $S_{1}$, the optimal solution can be easily achieved by modifying the associated zero-one variables only, while modifying both components of the solution will discard the optimal ordered list and lead to an ineffective search.

The aforementioned example demonstrates that permuting only the zero-one variable of an infeasible solution might be more effective than modifying both components of it. In particular, those low cost infeasible solutions should be focused more during the search process, because a solution with low cost is more likely to contain the optimal ordered list (so that only the zero-one variables need to be readjusted). However, few search operators have been designed for this purpose. Instead, most existing approaches handle infeasible solutions using relatively standard methods that are available in the constrained optimization literature and tend to overlook the promising infeasible solutions. For example, the CARPET [9], VND [10], and TSA [17] simply define the objective function as a weighted sum of cost and constraint violation. By tuning the weights, the search process biases more toward the feasible solutions, and no effort is spent to examine the infeasible solutions. In [12] and [13], a genotype representation scheme is adopted. To obtain the final solution, a split operator, which does not generate infeasible solutions at all, needs to be applied. Thus, only feasible solutions are considered in this case, and all the infeasible solutions are discarded even without calculating the cost. In [16], Handa et al. also adopt the weighted sum of cost and constraint violation as the objective function. In addition, a repair operator is proposed to handle infeasible solutions. Given an infeasible solution, the repair operator first figures out the route that violates the capacity constraint the most in the solution. Then, a task is randomly chosen from those tasks served in this route, and it will be moved to another route that traverses the selected task by deadheading path. Otherwise, no change will be made. The repair process terminates when the aforementioned procedure has been repeated for a predefined number of times or the constraint violation has been reduced to zero. To the best of our knowledge, this is the only existing repair operator that is specifically designed in the context of CARP. Nevertheless, this repair operator always works on a part (a single route) of the solution rather than the whole solution itself. Hence, it searches in a rather local region around the infeasible solution, and it might not be able to fully exploit the useful information in the infeasible solution. Based on the consideration that the low cost infeasible solutions deserve to be examined more carefully, we propose our GRO.

\section{C. $G R O$}

As mentioned earlier, GRO is specifically designed to deal with the low cost infeasible solutions. Given such a solution, GRO preserves its ordered list and reassigns the zero-one variables to minimize the constraint violation. In other words, GRO seeks the optimal assignment of zero-one variables for a given ordered list. Such a repair process takes into account all routes involved in the solution, and thus, GRO can be viewed as a global operator. Suppose that we have an infeasible solution with $m$ routes, reassigning the zero-one variables can be formulated as the following problem:

$$
\begin{aligned}
& \min \sum_{i=1}^{m}\left(\max \left[\sum_{j=1}^{N} s_{j} \times x_{i j}-Q, 0\right]\right) \\
& \text { s.t. : } \sum_{i \in \Omega(j)} x_{i j}=1, \quad j=1,2, \ldots, N \\
& x_{i j}=0 \text { or } 1, \quad i=1,2, \ldots, m ; \quad j=1,2, \ldots, N
\end{aligned}
$$

where $N$ is the total number of tasks, and $s_{j}$ denotes the serving cost for task $j . x_{i j}$ is set to one if task $j$ is served in the route $i$ and is set to zero if otherwise. $\Omega(j)$ is defined as

$$
\Omega(j)=\{i \mid \operatorname{task} j \text { is traversed in route } i \text { in } S\} .
$$

Given the ordered list, constraints (11) and (12) guarantee that each task is served only once among the routes it is traversed.

Let $\left\{a_{1}, a_{2}, \ldots, a_{N}\right\}$ and $\left\{b_{1}, b_{2}, \ldots, b_{m}\right\}$ be sets of items and bins, respectively. The aforementioned problem can be viewed as a bin-packing problem, where the size of the item 


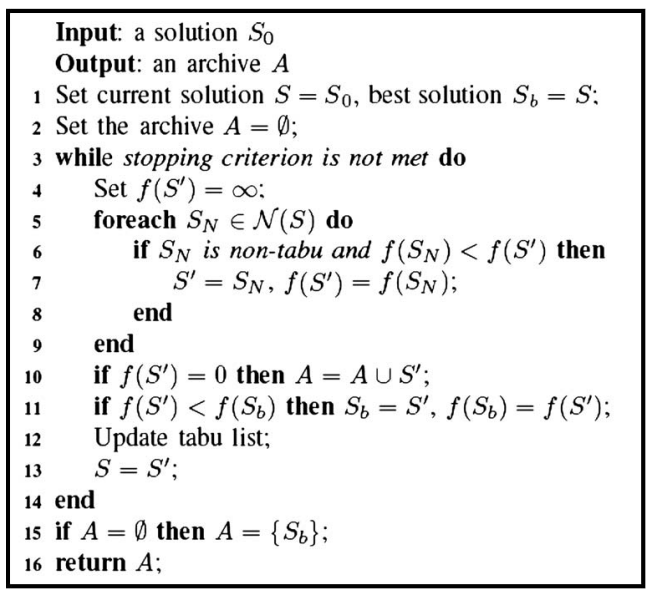

Fig. 3. Tabu search process.

$j$ is $s_{j}$, and all the bins share an identical capacity $Q$. GRO employs an insertion heuristic followed by a short-term tabu search to solve the bin-packing problem. The general idea of the insertion heuristic is straightforward. We sequentially pick an item out of the whole set and insert it into a bin, until all the items have been inserted. Such procedure can be described as follows.

Step 1) Initialize $x_{i j}=0, \forall i, j$. Let $A=\{1,2, \ldots, N\}$ and $c l\left(b_{i}\right)=0, \forall i$. Here, $\operatorname{cl}\left(b_{i}\right)$ is the current load of $b_{i}$. Then, repeat steps 2) to 4) until $A=\emptyset$.

Step 2) For each $j \in A$, identify the set $\bar{\Omega}(j)$ satisfying $\bar{\Omega}(j)=\left\{i \in \Omega(j) \mid c l\left(b_{i}\right)+s_{j} \leqslant Q\right\}$. Select the item corresponding to the smallest $|\bar{\Omega}(j)|$ as the one to be inserted. If multiple items share the smallest $|\bar{\Omega}(j)|$, the one with the largest $s_{j}$ will be selected. Then, ties are broken by selecting the item with the smallest index $(j)$. Selecting the items in this way guarantees that the item with the least choice of insertion without violating the constraints is chosen first.

Step 3) Identify the $b_{i}$ with the smallest $c l\left(b_{i}\right)$. If more than one bin has the smallest $c l\left(b_{i}\right)$, the one with the smallest

$$
\sum_{j \in A} I_{\Omega(j)}(i) \times s_{j}
$$

is selected, where $I_{\Omega(j)}(i)$ is an indicator function. $I_{\Omega(j)}(i)=1$ if $i \in \Omega(j)$ and zero if otherwise. The aforementioned equation indicates that the bin available for the least untreated items is considered first. After that, ties are broken by selecting the bin with the smallest index $(i)$.

Step 4) Insert the selected item $a_{j^{\prime}}$ in the chosen bin $b_{i^{\prime}}$. Set $x_{i^{\prime} j^{\prime}}=1$, remove $j^{\prime}$ from $A$, and update $c l\left(b_{i^{\prime}}\right)$ with $s_{j^{\prime}}$.

After obtaining the initial solution with the insertion heuristic, a standard tabu search is employed to further improve it, as shown in Fig. 3.

In Fig. 3, $S_{0}$ is the solution obtained by the insertion heuristic, and $f(S)$ denotes the objective function (10). The neighbor- hood $N(S)$ is a set of neighbors generated by moving one item to another admissible bin. The tabu list and aspiration criterion employed here are conventional. That is, an item that just leaves a bin is not allowed to return to that bin within a certain number of iterations (i.e., the tabu tenure), unless the resultant solution is better than the best solution found so far. The tabu tenure is set to $F / 2$, where $F$ is the number of items having more than one admissible bins. The tabu search terminates after $N$ iterations or consecutive $N / 3$ iterations without improvement.

Based on a solution of the bin-packing problem, a new solution of CARP can be directly obtained by updating the zero-one variables according to $x_{i j}$ 's. Since the ordered list remains unchanged when solving the bin-packing problem, the issue of cost is actually not considered at this stage. However, the tabu search process might generate different assignments of zero-one variables that all correspond to feasible solutions of the CARP, and it will be difficult to select them if the cost is not considered. Hence, we further define an archive in the tabu search process. As can be seen from line 10 in Fig. 3, at each iteration of the tabu search process, if the obtained best assignment of zero-one variables corresponds to a feasible solution to CARP, it will be included into the archive. After the termination of the tabu search process, all candidates in the archive will be fed into the following further refinement procedure to complete the repair process.

In the vertex encoding, two adjacent services of tasks are connected by the shortest path between them. When a new solution is obtained by changing the zero-one variables only, the path between two adjacent services might no longer be the shortest path. The new solution can be easily improved by a further refinement procedure, which modifies the ordered list of the solution without changing the zero-one variables so that any two adjacent services are connected with the shortest path between them. Hence, as the final step of our repair process, all the archived assignments of zero-one variables are transformed to the corresponding solutions of CARP; then, the ordered lists of these solutions are refined by updating the vertices between each pair of adjacent services with the shortest path. Finally, the solution with the lowest cost is chosen as the output of the repair process.

To summarize, the major steps of GRO are listed as follows.

1) Formulate the problem given by (10) as a bin-packing problem, and get a solution via the insertion heuristic.

2) Utilize a tabu search process to further improve the solution obtained in step 1), and get an archive of candidate assignments of zero-one variables.

3) Obtain new solutions of CARP based on the archived assignments of zero-one variables, and update these solutions with the further refinement procedure. The solution with the lowest cost is chosen as the output of GRO.

\section{EMBEDDING GRO IN TSA}

It can be observed that GRO is solely based on the vertex encoding. Hence, it can be embedded in any search algorithm that adopts the same encoding scheme. In this section, we demonstrate how to combine GRO with a recently proposed tabu search method. Belonging to the family of local search 
techniques, tabu search has been widely used to solve various real-world problems [18], [19]. The TSA employed here was specifically proposed for CARP [17].

In TSA, the function to be optimized is defined as a weighted sum of the cost and capacity violation, i.e., $f(S)=c(S)+$ $p \times c v(S)$, where $c(S)$ is the cost of $S$ and $c v(S)$ is the capacity violation of $S$. The self-adaptive parameter $p$ controls the tradeoff between the cost and feasibility. $p$ is set to one first and is halved (doubled) if all the solutions are feasible (infeasible) for ten consecutive iterations. TSA employs three move operators, namely, SI, DI, and swap1. During the search process, TSA realizes different search biases by adjusting the frequencies of applying the three operators $\left(F_{\mathrm{SI}}, F_{\mathrm{DI}}\right.$, and $\left.F_{\text {swap }}\right)$. Moreover, the Frederickson's heuristic [20] is also used to obtain further improvements. Since TSA is only used to demonstrate the efficacy of GRO and no modification is made to it throughout this work, we do not present full details of TSA in this paper. Interested readers are referred to the original publication [17].

In [17], the aforementioned TSA is named TSA version 1 (TSA1). Furthermore, a TSA version 2 (TSA2) has also been proposed. Generally speaking, TSA2 applies TSA1 to five different initial solutions simultaneously. After that, TSA1 is further applied to the best solution that has been achieved but with different parameter values. Experimental studies showed that TSA1 is much faster than three compared methods, namely, CARPET, MA, and TSA2, while still obtained acceptable solutions (better than CARPET and slightly worse than MA). The runtime of TSA2 is about five times more than TSA1, while it performs much better in terms of the quality of solutions.

The GRO is embedded in TSA with little effort. That is, every time an infeasible solution with the lowest cost so far is obtained, GRO is applied to it. We name such a simple combination of GRO and TSA1 as the RTS algorithm. Since both GRO and TSA1 are deterministic, RTS is a deterministic algorithm.

\section{EXPERIMENTAL STUDIES}

To evaluate the efficacy of GRO, we experimentally compare RTS to a number of state-of-the-art approaches in this section.

\section{A. Experimental Setup}

The experiments were carried out on five benchmark test sets of CARP problems, referred to as the $g d b, v a l$, and $e g l$ sets, the set of Beullens et al., and Brandão and Eglese's set. All these test sets have been studied in the literature. The $g d b$ set was generated by DeArmon in [21] and consisted of 23 instances. The val set was generated by Benavent et al. in [22]. It contains 34 instances based on ten different graphs. Different instances based on each graph were generated by changing the capacity of the vehicles. The $e g l$ set was generated by Eglese based on data from a winter gritting application in Lancashire [23]-[25]. It consists of 24 instances based on two graphs, each with a distinct set of required edges and capacity constraints. The test set generated by Beullens et al. in [14] is based on the intercity road network in Flanders. It further contains four subsets, namely, the sets $C, D, E$, and $F$, each of which contains 25 instances. The instances of sets $D$ and $F$ share the same networks with the instances of sets $C$ and $E$, respectively, but with larger capacity of vehicles. Generated by Brandão and Eglese in [17], the final test set consists of ten large instances defined on a road network with 255 vertices and 375 edges in Lancashire. Different instances in this set were created by changing the set of edges required for service and the capacity of the vehicles.

We considered six existing approaches in the comparative study. That is, the CARPET [9], VND [10], GLS [14], MA [12], TSA1, and TSA2 [17]. To facilitate the comparison between RTS and TSA1, RTS was implemented using the same parameters suggested for TSA1 in [17]. Since TSA2 applies TSA1 for six times, it might be inappropriate to directly compare RTS to TSA2. Instead, we further consider an extended version of RTS, represented by RTS* hereinafter. The RTS* was obtained by adjusting the stopping criterion of RTS, so that its runtime is increased by about four times. Except for the stopping criterion, RTS* adopts exactly the same parameters as RTS. In this way, we are able to conduct a more comprehensive comparison between RTS and the other approaches.

\section{B. Experimental Results}

Tables I-VIII present the experimental results of the compared approaches on all the five test sets. It should be noted that few existing algorithms have been tested on all the five benchmark test sets. On the $g d b$ and $v a l$ sets, the results of CARPET, VND, MA, TSA1, TSA2, RTS, and RTS* are available (Tables I and II). As shown in Table III, MA, TSA1, TSA2, RTS, and RTS $^{*}$ had been applied to the egl set. Comparison between GLS, TSA1, TSA2, RTS, and RTS* can be made based on the set of Beullens et al.; results for the four subsets are presented in Tables IV-VII. Finally, RTS and RTS* are only compared with TSA1 on the Brandão and Eglese's set (Table VIII), since no other algorithm has been applied to this set before. A brief description of the contents in each table is listed as follows.

1) The columns headed " $|\mathrm{V}|$, , “ $|\mathrm{R}|$," and “ $|\mathrm{E}|$ ” indicate the number of vertices, required edges, and total edges, respectively. Since all edges are required to be served in the $g d b$ set, the column $|\mathrm{R}|$ is omitted from Table I.

2) The column headed "LB" gives the lower bounds found so far for the problems, which are available in [14], [17], and [26]-[29]. Note that this column is absent in Table VIII because the lower bounds of the problems in the Brandão and Eglese's set are not available in the literature.

3) The columns headed "Cost" present the cost of the final solutions obtained by the corresponding algorithm. The columns headed "CPU(s)" provide the runtime (in CPU seconds) needed to obtain the solution.

4) For Tables I-VII, three additional rows are included at the bottom of the tables. The first row presents the average costs of solutions and runtimes calculated for each approach over all the instances in each set. The average values of lower bounds have also been calculated 
TABLE I

Results For gdb Benchmark Test SEt. "mean", "No.opt", AND “APD” Stand For Average Cost and Runtimes, NUMBER OF OPTIMAL SOLUTIONS, AND APD TO THE LOWER BOUNDS, RESPECTIVELY

\begin{tabular}{|c|c|c|c|c|c|c|c|c|c|c|c|c|c|c|c|c|c|}
\hline Name & $|\mathrm{V}|$ & $|E|$ & LB & $\begin{array}{r}\text { CI } \\
\text { Cost }\end{array}$ & $\begin{array}{l}\text { ARPET } \\
\mathrm{CPU}(\mathrm{s})\end{array}$ & Cost & $\begin{array}{l}\text { VND } \\
\text { CPU (s) }\end{array}$ & Cost & $\begin{array}{l}\mathrm{MA} \\
\mathrm{CPU}(\mathrm{s})\end{array}$ & Cost & $\begin{array}{l}\text { TSA1 } \\
\text { CPU (s) }\end{array}$ & Cost & $\begin{array}{l}\mathrm{TSA} 2 \\
\mathrm{CPU}(\mathrm{s})\end{array}$ & Cost & $\begin{array}{l}\text { RTS } \\
\text { CPU (s) }\end{array}$ & Cost & $\begin{array}{l}\operatorname{RTS}^{*} \\
\operatorname{CPU}(\mathrm{s})\end{array}$ \\
\hline 1 & 12 & 22 & 316 & 316 & 1.7 & 316 & 0.0 & 316 & 0.0 & 316 & 0.0 & 316 & 0.0 & 316 & 0.0 & 316 & 0.0 \\
\hline 2 & 12 & 26 & 339 & 339 & 2.8 & 339 & 0.0 & 339 & 0.2 & 345 & 0.1 & 339 & 0.1 & 339 & 0.0 & 339 & 0.0 \\
\hline 3 & 12 & 22 & 275 & 275 & 0.0 & 275 & 0.0 & 275 & 0.0 & 275 & 0.0 & 275 & 0.0 & 275 & 0.1 & 275 & 0.1 \\
\hline 4 & 11 & 19 & 287 & 287 & 0.1 & 287 & 0.0 & 287 & 0.0 & 287 & 0.0 & 287 & 0.0 & 287 & 0.0 & 287 & 0.0 \\
\hline 5 & 13 & 26 & 377 & 377 & 3.0 & 377 & 0.2 & 377 & 0.1 & 377 & 0.0 & 377 & 0.1 & 377 & 0.0 & 377 & 0.0 \\
\hline 6 & 12 & 22 & 298 & 298 & 0.5 & 298 & 0.0 & 298 & 0.1 & 298 & 0.0 & 298 & 0.0 & 298 & 0.0 & 298 & 0.0 \\
\hline 7 & 12 & 22 & 325 & 325 & 0.0 & 325 & 0.0 & 325 & 0.1 & 325 & 0.0 & 325 & 0.0 & 325 & 0.0 & 325 & 0.0 \\
\hline 8 & 27 & 46 & 348 & 352 & 33.0 & 350 & 5.2 & 350 & 18.6 & 352 & 1.8 & 348 & 1.1 & 348 & 2.0 & 348 & 9.4 \\
\hline 9 & 27 & 51 & 303 & 317 & 29.2 & 315 & 8.6 & 303 & 3.5 & 307 & 0.7 & 303 & 18.3 & 303 & 1.3 & 303 & 1.3 \\
\hline 10 & 12 & 25 & 275 & 275 & 0.8 & 275 & 0.0 & 275 & 0.0 & 275 & 0.0 & 275 & 0.0 & 275 & 0.0 & 275 & 0.0 \\
\hline 11 & 22 & 45 & 395 & 395 & 1.2 & 395 & 0.0 & 395 & 0.6 & 395 & 0.1 & 395 & 0.1 & 395 & 0.2 & 395 & 0.2 \\
\hline 12 & 13 & 23 & 458 & 458 & 11.2 & 458 & 1.6 & 458 & 4.9 & 462 & 0.1 & 458 & 0.6 & 458 & 1.7 & 458 & 1.7 \\
\hline 13 & 10 & 28 & 536 & 544 & 1.3 & 544 & 1.1 & 536 & 3.7 & 544 & 0.1 & 540 & 3.4 & 544 & 1.0 & 536 & 1.0 \\
\hline 14 & 7 & 21 & 100 & 100 & 0.3 & 100 & 0.0 & 100 & 0.0 & 100 & 0.0 & 100 & 0.1 & 100 & 0.0 & 100 & 0.0 \\
\hline 15 & 7 & 21 & 58 & 58 & 0.0 & 58 & 0.0 & 58 & 0.0 & 58 & 0.0 & 58 & 0.0 & 58 & 0.0 & 58 & 0.0 \\
\hline 16 & 8 & 28 & 127 & 127 & 0.9 & 127 & 0.2 & 127 & 0.0 & 129 & 0.1 & 127 & 0.1 & 127 & 0.0 & 127 & 0.0 \\
\hline 17 & 8 & 28 & 91 & 91 & 0.0 & 91 & 0.0 & 91 & 0.0 & 91 & 0.0 & 91 & 0.0 & 91 & 0.0 & 91 & 0.0 \\
\hline 18 & 9 & 36 & 164 & 164 & 0.1 & 164 & 0.0 & 164 & 0.1 & 164 & 0.0 & 164 & 0.0 & 164 & 0.3 & 164 & 0.3 \\
\hline 19 & 8 & 11 & 55 & 55 & 0.1 & 55 & 0.0 & 55 & 0.0 & 55 & 0.0 & 55 & 0.0 & 55 & 0.0 & 55 & 0.0 \\
\hline 20 & 11 & 22 & 121 & 121 & 5.2 & 121 & 0.1 & 121 & 0.2 & 123 & 0.1 & 121 & 0.1 & 121 & 0.0 & 121 & 0.0 \\
\hline 21 & 11 & 33 & 156 & 156 & 0.6 & 156 & 0.1 & 156 & 0.1 & 156 & 0.0 & 156 & 0.0 & 156 & 0.1 & 156 & 0.1 \\
\hline 22 & 11 & 44 & 200 & 200 & 1.8 & 200 & 0.8 & 200 & 1.7 & 200 & 0.0 & 200 & 0.1 & 200 & 0.0 & 200 & 0.0 \\
\hline 23 & 11 & 55 & 233 & 235 & 18.6 & 235 & 2.8 & 233 & 25.6 & 235 & 0.5 & 235 & 15.6 & 233 & 0.1 & 233 & 0.1 \\
\hline mean & & & 253.8 & 255.0 & 4.9 & 254.8 & 0.5 & 253.9 & 2.6 & 255.2 & 0.2 & 254.0 & 1.7 & 254.1 & 0.2 & 253.8 & 0.6 \\
\hline No. opt & & & 23 & 19 & - & 19 & - & 22 & - & 15 & - & 21 & - & 22 & - & 23 & - \\
\hline $\mathrm{APD}$ & & & - & 0.35 & - & 0.30 & - & 0.03 & - & 0.46 & - & 0.07 & - & 0.06 & - & 0.00 & - \\
\hline
\end{tabular}

for reference. The second row summarizes the number of instances on which the approach has achieved the optimal solutions (i.e., reaches the lower bounds). The third row calculates for each approach the average percentage deviation (APD) to the lower bounds. Because lower bounds are not available for the instances of the Brandão and Eglese's test set, only the average costs and runtimes are provided for Table VIII.

5) In all the tables, results are highlighted in bold for the instances on which RTS or RTS* achieved the optimal solutions.

In our experiments, RTS and RTS* were coded with C language and run using an Intel(R) Xeon(R) E5335 2.00 GHz. The results of the six existing approaches were directly obtained from the original publications. Since the compared approaches were implemented on different computers, normalization has been carried out to make fair comparisons on the runtimes. That is, all the runtimes presented in this paper were obtained via dividing the runtimes in the original publications by some factors. To be specific, CARPET and VND were implemented on the Graphics Indigo2 (195 MHz); thereby, the runtimes presented in [9] and [10] have been divided by ten. The results of GLS in [14] were obtained using a Pentium II $500 \mathrm{MHz}$; thus, we divided the runtimes there by four. The MA was implemented on a Pentium III $1 \mathrm{GHz}$ and the TSA on a Pentium Mobile $1.4 \mathrm{GHz}$. Hence, the runtimes of MA, TSA1, and TSA2 given in the corresponding papers have been divided by $2,10 / 7$, and $10 / 7$, respectively.

The efficacy of GRO can be evaluated from two perspectives, i.e., the quality of solution and the computational time. The average cost, the number of optimal solutions achieved, and the APD are all examined to get a more comprehensive comparison on the quality of solution. From Tables I and II, it can be seen that MA, TSA2, RTS, and RTS* performed comparably on the $g d b$ and $v a l$ sets, while the results of CARPET, VND, and TSA1 are inferior. Among the former four methods, RTS requires the least runtime, and RTS* is more efficient than MA and TSA2.

On the egl set, RTS* significantly outperformed the other methods in terms of solution qualities. MA, TSA2, and RTS achieved comparable results, and TSA1 performed the worst. Furthermore, RTS* is less time consuming than MA and TSA2, not to mention RTS.

In [14] and [17], results on the four test subsets of Beullens et al. were reported in terms of the cost of deadheading only. Hence, we present the results of RTS and RTS* in the same form in Tables IV-VII. In general, GLS exhibited the best overall performance, followed by RTS*, TSA2, and RTS. However, we can further find that GLS outperformed the RTS* marginally, while the runtime of RTS* is always shorter than GLS. This is particularly obvious on sets $C$ and $E$. RTS* appears to be superior to TSA2 on set $F$, and the two achieved similar performance on sets $C, D$, and $E$. On the other hand, RTS* requires about half of the runtime of TSA2 on all the four sets. Unsurprisingly, TSA1 did not perform as good as the others.

Finally, as shown in Table VIII, RTS not only achieved solutions of higher qualities than TSA1 but also was computationally more efficient. Since only the results of TSA1 are available in the literature, the results of RTS* are presented for reference only.

To summarize, RTS and RTS* are competitive with a number of state-of-the-art approaches for CARP. In particular, RTS is the most efficient one among all the compared approaches, and it also managed to achieve high quality solutions on many 
TABLE II

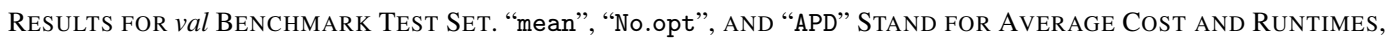
Number of OPTIMAL SOLUTIONS, AND APD TO THE LOWER BOUNDS, RESPECTIVELY

\begin{tabular}{|c|c|c|c|c|c|c|c|c|c|c|c|c|c|c|c|c|c|}
\hline Name & $|\mathrm{V}|$ & $|\mathrm{E}|$ & LB & $\begin{array}{r}C F \\
\text { Cost }\end{array}$ & $\begin{array}{l}\operatorname{RPET} \\
\mathrm{CPU}(\mathrm{S})\end{array}$ & Cost & $\begin{array}{l}\text { VND } \\
\mathrm{CPU}(\mathrm{s})\end{array}$ & Cost & $\begin{array}{l}\mathrm{MA} \\
\mathrm{CPU}(\mathrm{s})\end{array}$ & Cost & $\begin{array}{l}\text { CSA1 } \\
\text { CPU (s) }\end{array}$ & Cost & $\begin{array}{l}\text { ISA2 } \\
\mathrm{CPU}(\mathrm{s})\end{array}$ & Cost & $\begin{array}{l}\text { RTS } \\
\operatorname{CPU}(S)\end{array}$ & Cost & $\begin{array}{l}\operatorname{RTS}^{*} \\
\operatorname{CPU}(\mathrm{s})\end{array}$ \\
\hline $1 \mathrm{~A}$ & 24 & 39 & 173 & 173 & 0.0 & 173 & 0.0 & 173 & 0.0 & 173 & 0.0 & 173 & 0.0 & 173 & 0.0 & 173 & 0.0 \\
\hline $1 \mathrm{~B}$ & 24 & 39 & 173 & 173 & 5.0 & 173 & 0.4 & 173 & 4.0 & 173 & 0.1 & 173 & 0.6 & 173 & 0.5 & 173 & 0.5 \\
\hline $1 \mathrm{C}$ & 24 & 39 & 245 & 245 & 50.6 & 245 & 1.6 & 245 & 14.3 & 245 & 0.6 & 245 & 8.5 & 245 & 0.0 & 245 & 0.0 \\
\hline $2 \mathrm{~A}$ & 24 & 34 & 227 & 227 & 0.1 & 227 & 0.0 & 227 & 0.0 & 227 & 0.0 & 227 & 0.0 & 227 & 0.0 & 227 & 0.0 \\
\hline $2 B$ & 24 & 34 & 259 & 260 & 7.1 & 259 & 0.2 & 259 & 0.1 & 259 & 0.1 & 259 & 0.2 & 259 & 0.1 & 259 & 0.1 \\
\hline $2 \mathrm{C}$ & 24 & 34 & 457 & 494 & 17.2 & 457 & 3.4 & 457 & 10.9 & 457 & 1.0 & 457 & 5.5 & 457 & 0.3 & 457 & 0.3 \\
\hline $3 \mathrm{~A}$ & 24 & 35 & 81 & 81 & 0.4 & 81 & 0.0 & 81 & 0.0 & 81 & 0.0 & 81 & 0.0 & 81 & 0.0 & 81 & 0.0 \\
\hline $3 B$ & 24 & 35 & 87 & 87 & 1.5 & 87 & 0.0 & 87 & 0.0 & 87 & 0.0 & 87 & 0.0 & 87 & 0.0 & 87 & 0.0 \\
\hline $3 C$ & 24 & 35 & 138 & 138 & 22.6 & 140 & 2.7 & 138 & 14.1 & 138 & 0.4 & 138 & 0.9 & 138 & 0.2 & 138 & 0.2 \\
\hline $4 \mathrm{~A}$ & 41 & 69 & 400 & 400 & 15.4 & 400 & 0.1 & 400 & 0.4 & 400 & 0.1 & 400 & 0.3 & 400 & 0.0 & 400 & 0.0 \\
\hline $4 B$ & 41 & 69 & 412 & 416 & 41.0 & 414 & 2.0 & 412 & 0.6 & 414 & 1.2 & 412 & 3.9 & 412 & 0.9 & 412 & 0.9 \\
\hline $4 \mathrm{C}$ & 41 & 69 & 428 & 453 & 38.0 & 428 & 3.9 & 428 & 9.6 & 444 & 1.2 & 428 & 26.6 & 428 & 4.6 & 428 & 4.6 \\
\hline $4 \mathrm{D}$ & 41 & 69 & 526 & 556 & 126.6 & 544 & 6.6 & 541 & 51.6 & 538 & 7.2 & 530 & 77.0 & 530 & 1.3 & 530 & 21.0 \\
\hline $5 \mathrm{~A}$ & 34 & 65 & 423 & 423 & 2.1 & 423 & 0.1 & 423 & 0.9 & 423 & 0.2 & 423 & 0.2 & 423 & 0.4 & 423 & 0.4 \\
\hline $5 B$ & 34 & 65 & 446 & 448 & 22.4 & 449 & 1.4 & 446 & 0.1 & 446 & 0.1 & 446 & 0.1 & 446 & 0.0 & 446 & 0.0 \\
\hline $5 \mathrm{C}$ & 34 & 65 & 473 & 476 & 28.9 & 474 & 2.4 & 474 & 50.5 & 474 & 0.8 & 474 & 7.4 & 474 & 1.0 & 474 & 21.0 \\
\hline 5D & 34 & 65 & 573 & 607 & 121.5 & 599 & 4.2 & 583 & 45.4 & 583 & 4.6 & 583 & 51.3 & 583 & 4.8 & 583 & 24.3 \\
\hline $6 \mathrm{~A}$ & 31 & 50 & 223 & 223 & 2.11 & 223 & 0.0 & 223 & 0.1 & 223 & 0.1 & 223 & 1.1 & 223 & 0.0 & 223 & 0.0 \\
\hline $6 \mathrm{~B}$ & 31 & 50 & 233 & 241 & 14.6 & 233 & 1.2 & 233 & 33.7 & 233 & 1.8 & 233 & 8.9 & 233 & 0.5 & 233 & 0.5 \\
\hline $6 \mathrm{C}$ & 31 & 50 & 317 & 329 & 46.2 & 325 & 4.2 & 317 & 26.1 & 323 & 2.2 & 317 & 16.0 & 317 & 0.0 & 317 & 0.0 \\
\hline $7 \mathrm{~A}$ & 40 & 66 & 279 & 279 & 3.6 & 279 & 0.0 & 279 & 0.9 & 283 & 0.6 & 279 & 0.7 & 279 & 1.2 & 279 & 1.2 \\
\hline $7 \mathrm{~B}$ & 40 & 66 & 283 & 283 & 0.0 & 283 & 0.0 & 283 & 0.2 & 283 & 0.1 & 283 & 0.4 & 283 & 0.9 & 283 & 0.9 \\
\hline $7 \mathrm{C}$ & 40 & 66 & 334 & 343 & 65.8 & 335 & 4.5 & 334 & 50.6 & 335 & 2.8 & 334 & 25.9 & 334 & 3.1 & 334 & 3.1 \\
\hline $8 \mathrm{~A}$ & 30 & 63 & 386 & 386 & 2.1 & 386 & 0.2 & 386 & 0.3 & 386 & 0.4 & 386 & 0.2 & 386 & 0.1 & 386 & 0.1 \\
\hline $8 B$ & 30 & 63 & 395 & 401 & 44.2 & 403 & 1.1 & 395 & 5.0 & 407 & 0.7 & 395 & 1.3 & 395 & 0.0 & 395 & 0.0 \\
\hline $8 \mathrm{C}$ & 30 & 63 & 518 & 533 & 79.9 & 543 & 5.6 & 527 & 35.7 & 545 & 1.3 & 529 & 39.0 & 545 & 1.5 & 524 & 20.0 \\
\hline $9 \mathrm{~A}$ & 50 & 92 & 323 & 323 & 15.5 & 323 & 1.4 & 323 & 9.1 & 323 & 0.5 & 323 & 0.0 & 323 & 1.5 & 323 & 1.5 \\
\hline $9 B$ & 50 & 92 & 326 & 329 & 32.5 & 326 & 2.6 & 326 & 14.7 & 326 & 0.9 & 326 & 0.4 & 326 & 2.5 & 326 & 2.5 \\
\hline $9 \mathrm{C}$ & 50 & 92 & 332 & 332 & 30.6 & 336 & 2.8 & 332 & 35.6 & 332 & 0.5 & 332 & 0.3 & 332 & 4.0 & 332 & 4.0 \\
\hline $9 \mathrm{D}$ & 50 & 92 & 385 & 409 & 191.5 & 399 & 6.2 & 391 & 105.6 & 404 & 5.1 & 391 & 42.3 & 391 & 9.1 & 391 & 54.7 \\
\hline $10 \mathrm{~A}$ & 50 & 97 & 428 & 428 & 3.0 & 428 & 0.2 & 428 & 12.7 & 430 & 2.5 & 428 & 2.2 & 428 & 3.6 & 428 & 3.6 \\
\hline $10 \mathrm{~B}$ & 50 & 97 & 436 & 436 & 10.0 & 436 & 0.8 & 436 & 2.3 & 438 & 2.5 & 436 & 1.3 & 436 & 0.4 & 436 & 0.4 \\
\hline $10 \mathrm{C}$ & 50 & 97 & 446 & 451 & 50.7 & 446 & 3.3 & 446 & 8.7 & 447 & 3.2 & 446 & 5.3 & 446 & 5.0 & 446 & 5.0 \\
\hline $10 \mathrm{D}$ & 50 & 97 & 525 & 544 & 84.7 & 538 & 7.7 & 530 & 107.5 & 534 & 7.6 & 530 & 152.7 & 534 & 12.4 & 534 & 61.2 \\
\hline mean & & & 343.2 & 350.8 & 34.6 & 347.5 & 2.1 & 345.2 & 21.6 & 347.5 & 1.5 & 344.9 & 14.1 & 345.5 & 1.8 & 344.9 & 6.8 \\
\hline No.opt & & & & 17 & - & 21 & - & 28 & - & 19 & - & 28 & - & 28 & - & 28 & - \\
\hline APD & & & - & 1.60 & - & 0.82 & - & 0.27 & - & 0.77 & - & 0.22 & - & 0.33 & - & 0.21 & - \\
\hline
\end{tabular}

problems. Although RTS* is computationally more expensive than RTS, it provided significantly better solutions. Furthermore, RTS* is still much less time consuming than those compared methods that perform similarly to RTS* in terms of quality of solutions. Hence, RTS* can be said to give a good tradeoff between quality and time.

Since we are proposing a repair operator (i.e., the GRO) rather than an algorithm (i.e., the RTS) in this paper, it is worthwhile to study the role that the GRO plays in RTS. This can be done by comparing RTS and RTS* to TSA1 and TSA2, respectively. From the perspective of solution qualities, RTS outperformed TSA on 120 problem instances among a total of 191 problem instances investigated in this paper. RTS* outperformed TSA2 on 42 problem instances, while was inferior on eight only. The statistics presented in the previous tables also evidence the superiority of RTS and RTS*. Hence, the incorporation of GRO undoubtedly improves TSA1's performance.

Given the improvement brought by GRO, we further investigate the computational time required by GRO during the optimization process. For this purpose, we recorded the total runtime of RTS* and the time occupied by GRO in it. The average runtime on each test set and all the sets are presented in Table IX. We can see that GRO accounted for no more than $4 \%$ runtime of RTS*. Hence, it is reasonable to expect that the runtimes of RTS are slightly longer than that of TSA1. Nevertheless, as shown in Tables I-VIII, RTS is actually much less time consuming than TSA1. The reason is that the RTS converged faster than the TSA1 and thereby stopped earlier. Moreover, we also noticed that the difference between RTS and RTS* is marginal on quite a lot of problem instances. For example, the APDs of the two algorithms are very close to each other for the $g d b$ set, val set, and set $E$ of the set of Beullens et als. Such an observation further demonstrates the fast convergence of RTS.

\section{Further Analysis}

Since RTS* was outperformed by the compared methods on some instances, we attempt to further analyze when RTS* is preferable. The general idea is to find the correlation (if any) between the performance of RTS* and some measurable characteristics of the problem instances. Since there is no work regarding this issue so far, we define the following metric, namely, proportion of free tasks $(P F T)$, for our analysis. It is defined as follows:

$$
P F T=\frac{\sum_{(i, j) \in E_{R} \cup A_{R}} I_{(0, A R]} d(i, j)}{N}
$$


TABLE III

RESUltS FOR egl BENCHMARK TEST SET. "mean", "No.opt”, AND “APD” STAND FOR AVERAge Cost AND RUNTIMES, Number of Optimal SOLUtions, AND APD to the LOWER BOUndS, RESPECTIVELY

\begin{tabular}{|c|c|c|c|c|c|c|c|c|c|c|c|c|c|c|}
\hline \multirow[t]{2}{*}{ Name } & \multirow[t]{2}{*}{$|\mathrm{V}|$} & \multirow[t]{2}{*}{$|R|$} & \multirow[t]{2}{*}{$|E|$} & \multirow[t]{2}{*}{ LB } & \multicolumn{2}{|c|}{ MA } & \multicolumn{2}{|c|}{ TSA1 } & \multicolumn{2}{|c|}{ TSA2 } & \multicolumn{2}{|c|}{ RTS } & \multicolumn{2}{|c|}{ RTS $^{*}$} \\
\hline & & & & & Cost & $\mathrm{CPU}(\mathrm{s})$ & Cost & $\mathrm{CPU}(\mathrm{s})$ & Cost & $\mathrm{CPU}(\mathrm{s})$ & Cost & $\mathrm{CPU}(\mathrm{s})$ & Cost & $\mathrm{CPU}(\mathrm{s})$ \\
\hline $\mathrm{E} 1-\mathrm{A}$ & 77 & 51 & 98 & 3548 & 3548 & 37.1 & 3548 & 1.5 & 3548 & 15.5 & 3548 & 0.0 & 3548 & 0.0 \\
\hline $\mathrm{E} 1-\mathrm{B}$ & 77 & 51 & 98 & 4498 & 4498 & 34.7 & 4533 & 3.4 & 4533 & 19.6 & 4525 & 2.5 & 4498 & 8.8 \\
\hline E1-C & 77 & 51 & 98 & 5566 & 5595 & 35.6 & 5659 & 3.6 & 5595 & 16.9 & 5595 & 2.8 & 5595 & 13.5 \\
\hline $\mathrm{E} 2-\mathrm{A}$ & 77 & 72 & 98 & 5018 & 5018 & 76.3 & 5018 & 5.4 & 5018 & 44.4 & 5018 & 0.0 & 5018 & 0.0 \\
\hline$E 2-B$ & 77 & 72 & 98 & 6305 & 6340 & 76.7 & 6385 & 8.1 & 6343 & 46.7 & 6338 & 6.6 & 6317 & 29.2 \\
\hline $\mathrm{E} 2-\mathrm{C}$ & 77 & 72 & 98 & 8243 & 8415 & 64.8 & 8400 & 8.4 & 8347 & 55.1 & 8356 & 6.8 & 8335 & 32.6 \\
\hline E3-A & 77 & 87 & 98 & 5898 & 5898 & 121.0 & 6044 & 12.5 & 5902 & 54.1 & 5898 & 0.9 & 5898 & 0.9 \\
\hline E3-B & 77 & 87 & 98 & 7704 & 78 & 1 & 7916 & 1 & 7816 & 79.4 & 7799 & 10.9 & 7787 & 47.9 \\
\hline E3-C & 77 & 87 & 98 & 10163 & 10433 & 103.2 & 10309 & 16.2 & 10309 & 94.0 & 10326 & 10.9 & 10305 & 52.8 \\
\hline $\mathrm{E} 4-\mathrm{A}$ & 77 & 98 & 98 & 6408 & 6461 & 145.9 & 6476 & 9.8 & 6473 & 94.9 & 6464 & 11.7 & 6461 & 56.8 \\
\hline $\mathrm{E} 4-\mathrm{B}$ & 77 & 98 & 98 & 8884 & 9021 & 156.4 & 9134 & 18.8 & 9063 & 117.3 & 9026 & 12.7 & 9026 & 61.5 \\
\hline $\mathrm{E} 4-\mathrm{C}$ & 77 & 98 & 98 & 11427 & 11779 & 126.2 & 11627 & 22.3 & 11627 & 132.0 & 11598 & 16.9 & 11598 & 70.1 \\
\hline $\mathrm{S} 1-\mathrm{A}$ & 140 & 75 & 190 & 5018 & 50 & & 1 & .2 & 72 & 46.6 & 18 & 1. & 5018 & 1.7 \\
\hline$S 1-B$ & 140 & 75 & 19 & 6384 & 64 & 10 & 63 & 9.2 & 38 & 56.6 & 64 & 6. & 6394 & 32.5 \\
\hline $\mathrm{S} 1-\mathrm{C}$ & 140 & 75 & 190 & 8493 & 8518 & 82 & 873 & 4.8 & 8535 & 55.4 & 8518 & 7.4 & 8518 & 35.4 \\
\hline $\mathrm{S} 2-\mathrm{A}$ & 140 & 147 & 190 & 9824 & 9995 & 437.2 & 10190 & 49.1 & 10038 & 276.6 & 10087 & 37.8 & 9970 & 212.1 \\
\hline$S 2-B$ & 140 & 147 & 190 & 12968 & 13174 & 380.3 & 13284 & 54.7 & 13178 & 313.8 & 13345 & 42.8 & 13345 & 207.7 \\
\hline $\mathrm{S} 2-\mathrm{C}$ & 140 & 147 & 190 & 16353 & 16 & & & 37.5 & & & & & 00 & 227.8 \\
\hline S3-A & 140 & 159 & 190 & 10143 & 10296 & 535.3 & 10508 & 55.5 & 10451 & 387.9 & 10284 & 46.4 & 10284 & 221.5 \\
\hline$S 3-B$ & 140 & 159 & 190 & 13616 & 14053 & 532.0 & 13981 & 58.9 & 13981 & 399.4 & 13979 & 50.5 & 13857 & 242.6 \\
\hline S3-C & 140 & 159 & 190 & 17100 & 17297 & 437.2 & 17346 & 69.4 & 17346 & 417.5 & 17497 & 53.9 & 17316 & 264.4 \\
\hline $\mathrm{S} 4-\mathrm{A}$ & 140 & 190 & 190 & 12143 & 12442 & 768.8 & 12546 & 90.9 & 12462 & 487.8 & 12388 & 74.5 & 12348 & 355.0 \\
\hline$S 4-B$ & 140 & 190 & 190 & 16093 & 16531 & 715.1 & 16695 & 99.0 & 16490 & 668.2 & 16612 & 91. & 16442 & 406.9 \\
\hline $\mathrm{S} 4-\mathrm{C}$ & 140 & 190 & 190 & 20375 & 20832 & 747.5 & 20981 & 101.4 & 20733 & 654.2 & 21156 & 91.2 & 20821 & 437.2 \\
\hline mean & & & & 9673.8 & 9842.3 & 263.5 & 9899.5 & 31.7 & 9823.0 & 204.0 & 9852.6 & 26.4 & 9804.1 & 125.8 \\
\hline No. opt & & & & & 5 & - & 2 & - & 2 & 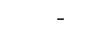 & 4 & - & 5 & - \\
\hline APD & & & & - & 1.38 & - & 2.08 & - & 1.30 & - & 1.43 & - & 1.04 & - \\
\hline
\end{tabular}

TABLE IV

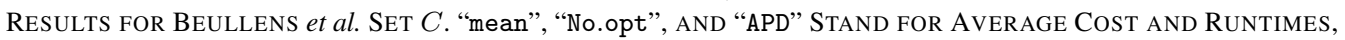
Number of OPTIMAL SOlutions, AND APD to THE LOWER BOUNDS, RESPECTIVELY

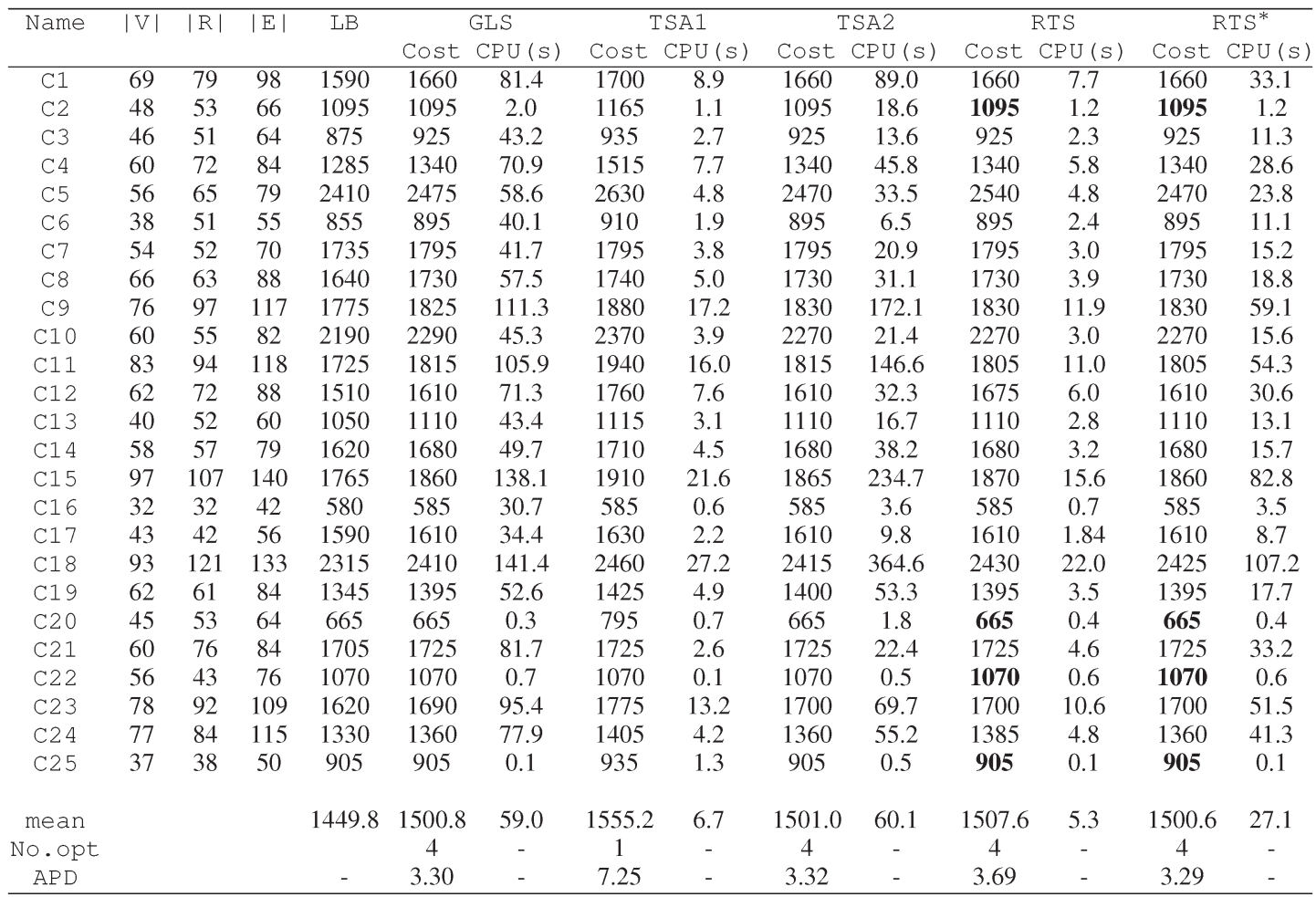

where

$$
A R=Q-\frac{\sum_{(i, j) \in E_{R} \cup A_{R}} d(i, j)}{\left\lceil\sum_{(i, j) \in E_{R} \cup A_{R}} d(i, j) / Q\right\rceil}
$$

$$
I_{(0, A R]} d(i, j)= \begin{cases}1, & \text { if } d(i, j) \leqslant A R \\ 0, & \text { otherwise. }\end{cases}
$$


TABLE V

RESUlts For Beullens et al. Set D. "mean", "No.opt", AND “APD” STAND For Average Cost And Runtimes, Number of Optimal SOLUTIONS, AND APD to THE LOWER BOUNDS, RESPECTIVELY

\begin{tabular}{|c|c|c|c|c|c|c|c|c|c|c|c|c|c|c|}
\hline Name & $|\mathrm{V}|$ & $|\mathrm{R}|$ & $|\mathrm{E}|$ & LB & & GLS & & [SA1 & & TSA2 & & RTS & & RTS* \\
\hline & & & & & Cost & $\mathrm{CPU}(\mathrm{s})$ & Cost & $\mathrm{CPU}(\mathrm{s})$ & Cost & $\mathrm{CPU}(\mathrm{s})$ & Cost & $\mathrm{CPU}(\mathrm{s})$ & Cost & $\mathrm{CPU}(\mathrm{s}$ \\
\hline D1 & 69 & 79 & 98 & 725 & 725 & 3.0 & 865 & 2.0 & 740 & 43.9 & 740 & 3.1 & 740 & 28.7 \\
\hline D2 & 48 & 53 & 66 & 480 & 480 & 0.3 & 480 & 1.1 & 480 & 0.6 & 480 & 0.3 & 480 & 0.3 \\
\hline D3 & 46 & 51 & 64 & 415 & 415 & 0.1 & 415 & 0.1 & 415 & 0.1 & 415 & 0.0 & 415 & 0.0 \\
\hline D4 & 60 & 72 & 84 & 615 & 615 & 0.1 & 630 & 3.1 & 615 & 1.2 & 615 & 0.1 & 615 & 0.1 \\
\hline D5 & 56 & 65 & 79 & 1040 & 1040 & 0.4 & 1080 & 1.7 & 1040 & 2.0 & 1040 & 1.1 & 1040 & 1.1 \\
\hline D6 & 38 & 51 & 55 & 485 & 485 & 0.1 & 525 & 0.5 & 485 & 5.5 & 485 & 0.7 & 485 & 0.7 \\
\hline D7 & 54 & 52 & 70 & 735 & 835 & 34.7 & 915 & 2.4 & 835 & 16.0 & 835 & 2.5 & 835 & 11.8 \\
\hline D8 & 66 & 63 & 88 & 615 & 685 & 48.8 & 685 & 3.2 & 685 & 34.4 & 685 & 3.3 & 685 & 16.5 \\
\hline D9 & 76 & 97 & 117 & 680 & 680 & 0.3 & 810 & 4.3 & 680 & 21.1 & 680 & 3.0 & 680 & 3.0 \\
\hline D10 & 60 & 55 & 82 & 900 & 910 & 37.3 & 910 & 0.9 & 910 & 11.6 & 910 & 1.4 & 910 & 10.1 \\
\hline D11 & 83 & 94 & 118 & 920 & 930 & 92.1 & 990 & 5.6 & 960 & 78.4 & 1000 & 3.2 & 930 & 41.3 \\
\hline D12 & 62 & 72 & 88 & 680 & 680 & 0.1 & 735 & 1.8 & 680 & 18.2 & 730 & 5.1 & 680 & 8.6 \\
\hline D13 & 40 & 52 & 60 & 690 & 690 & 0.2 & 695 & 0.8 & 695 & 9.7 & 690 & 0.3 & 690 & 0.3 \\
\hline D14 & 58 & 57 & 79 & 920 & 930 & 45.1 & 950 & 0.7 & 940 & 14.8 & 930 & 0.6 & 930 & 9.9 \\
\hline D15 & 97 & 107 & 140 & 910 & 910 & 92.2 & 1100 & 16.5 & 950 & 71.6 & 920 & 14.1 & 920 & 72.8 \\
\hline D16 & 32 & 32 & 42 & 170 & 170 & 0.0 & 175 & 0.1 & 170 & 0.3 & 170 & 0.0 & 170 & 0.0 \\
\hline D17 & 43 & 42 & 56 & 675 & 675 & 0.1 & 675 & 0.0 & 675 & 0.0 & 675 & 0.2 & 675 & 0.2 \\
\hline D18 & 93 & 121 & 133 & 930 & 930 & 0.6 & 1075 & 9.7 & 930 & 133.4 & 950 & 9.2 & 950 & 101.1 \\
\hline D19 & 62 & 61 & 84 & 650 & 680 & 38.7 & 690 & 1.9 & 690 & 14.1 & 690 & 0.6 & 680 & 13.1 \\
\hline D20 & 45 & 53 & 64 & 415 & 415 & 0.0 & 420 & 1.8 & 415 & 0.8 & 415 & 0.0 & 415 & 0.0 \\
\hline D21 & 60 & 76 & 84 & 695 & 805 & 63.0 & 865 & 1.6 & 825 & 42.6 & 815 & 6.2 & 810 & 27.0 \\
\hline D22 & 56 & 43 & 76 & 690 & 690 & 0.1 & 690 & 0.0 & 690 & 0.0 & 690 & 0.1 & 690 & 0.1 \\
\hline D23 & 78 & 92 & 109 & 715 & 735 & 83.8 & 780 & 3.7 & 735 & 77.8 & 755 & 3.8 & 735 & 45.0 \\
\hline D24 & 77 & 84 & 115 & 620 & 670 & 62.0 & 670 & 7.2 & 670 & 73.6 & 765 & 1.7 & 670 & 35.0 \\
\hline D25 & 37 & 38 & 50 & 410 & 410 & 0.0 & 510 & 0.1 & 410 & 0.4 & 410 & 0.0 & 410 & 0.0 \\
\hline mean & & & & 671.2 & 687.6 & 24.1 & 733.4 & 2.8 & 693.2 & 26.9 & 699.6 & 2.4 & 689.6 & 17.3 \\
\hline No.opt & & & & & 15 & - & 4 & - & 13 & . & 12 & - & 13 & - \\
\hline APD & & & & - & 2.38 & . & 8.90 & . & 3.02 & - & 4.04 & - & 2.62 & - \\
\hline
\end{tabular}

TABLE VI

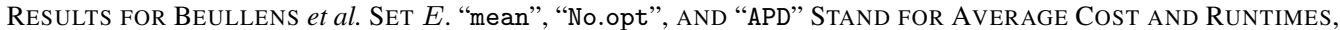
Number of OPTIMAL SOLUTIONS, AND APD to THE LOWER BOUNDS, RESPECTIVELY

\begin{tabular}{|c|c|c|c|c|c|c|c|c|c|c|c|c|c|c|}
\hline \multirow[t]{2}{*}{ Name } & \multirow[t]{2}{*}{$|V|$} & \multirow[t]{2}{*}{$|R|$} & \multirow[t]{2}{*}{$|E|$} & \multirow[t]{2}{*}{ LB } & \multicolumn{2}{|r|}{ GLS } & \multicolumn{2}{|c|}{ TSA1 } & \multicolumn{2}{|c|}{ TSA2 } & \multicolumn{2}{|r|}{ RTS } & \multicolumn{2}{|c|}{$\mathrm{RTS}^{*}$} \\
\hline & & & & & Cost & $\mathrm{CPU}(\mathrm{s})$ & Cost & $\mathrm{CPU}(\mathrm{s})$ & Cost & $\operatorname{CPU}(\mathrm{s})$ & Cost & $\mathrm{CPU}(\mathrm{s})$ & Cost & $\mathrm{CPU}(\mathrm{s})$ \\
\hline E1 & 73 & 85 & 105 & 1855 & 1940 & 85.5 & 2160 & 9.4 & 1935 & 142.2 & 1935 & 9.1 & 1935 & 45.4 \\
\hline E2 & 58 & 58 & 81 & 1580 & 1610 & 47.2 & 1700 & 3.9 & 1610 & 37.2 & 1610 & 3.4 & 1610 & 16.3 \\
\hline E4 & 70 & 77 & 99 & 1580 & 1610 & 82.1 & 1760 & 7.8 & 1615 & 92.6 & 1610 & 7.1 & 1610 & 36.6 \\
\hline E5 & 68 & 61 & 94 & 2130 & 2170 & 53.5 & 2235 & 6.0 & 2160 & 57.3 & 2185 & 3.6 & 2185 & 18.0 \\
\hline E 6 & 49 & 43 & 66 & 670 & 670 & 0.0 & 670 & 0.0 & 670 & 0.5 & 670 & 0.4 & 670 & 0.4 \\
\hline E8 & 74 & 59 & 98 & 2080 & 2150 & 55.4 & 2180 & 4.6 & 2155 & 58.6 & 2150 & 3.6 & 2150 & 17.7 \\
\hline E9 & 93 & 103 & 141 & 2160 & 2250 & 110.1 & 2440 & 17.0 & 2300 & 180.1 & 2325 & 15.0 & 2285 & 78.7 \\
\hline E10 & 56 & 49 & 76 & 1690 & 1690 & 0.1 & 1690 & 0.9 & 1690 & 2.6 & 1690 & 2.0 & 1690 & 2.0 \\
\hline E11 & 80 & 94 & 113 & 1810 & 1850 & 105.1 & 1980 & 15.7 & 1855 & 122.0 & 1850 & 12.7 & 1850 & 56.7 \\
\hline E12 & 74 & 67 & 103 & 1580 & 1710 & 66.2 & 1735 & 5.0 & 1730 & 62.8 & 1735 & 5.5 & 1715 & 25.9 \\
\hline E13 & 49 & 52 & 73 & 1300 & 1325 & 44.6 & 1415 & 0.7 & 1325 & 27.3 & 1325 & 2.6 & 1325 & 12.8 \\
\hline E18 & 78 & 88 & 110 & 1600 & 1610 & 90.8 & 1660 & 3.5 & 1610 & 86.2 & 1610 & 8.2 & 1610 & 49.9 \\
\hline E19 & 77 & 66 & 103 & 1400 & 1435 & 52.9 & 1475 & 5.5 & 1435 & 71.1 & 1435 & 4.8 & 1435 & 22.9 \\
\hline E20 & 56 & 63 & 80 & 950 & 990 & 58.1 & 1020 & 5.1 & 990 & 55.5 & 990 & 3.8 & 990 & 18.5 \\
\hline E21 & 57 & 72 & 82 & 1700 & 1705 & 73.4 & 1790 & 2.7 & 1705 & 54.9 & 1705 & 5.4 & 1705 & 26.8 \\
\hline E22 & 54 & 44 & 73 & 1155 & 1185 & 32.3 & 1215 & 2.0 & 1185 & 21.5 & 1185 & 1.5 & 1185 & 7.6 \\
\hline E23 & 93 & 89 & 130 & 1395 & 1435 & 98.7 & 1530 & 12.5 & 1445 & 132.7 & 1440 & 9.9 & 1430 & 49.4 \\
\hline E24 & 97 & 86 & 142 & 1695 & 1785 & 90.5 & 1850 & 9.4 & 1785 & 78.8 & 1785 & 2.8 & 1785 & 41.8 \\
\hline E2 5 & 26 & 28 & 35 & 655 & 655 & 0.0 & 655 & 0.1 & 655 & 0.1 & 655 & 0.1 & 655 & 0.1 \\
\hline mean & & & & 1517.0 & 1558.2 & 56.5 & 1619.6 & 5.6 & 1561.6 & 64.4 & 1563.6 & 4.9 & 1560.2 & 26.1 \\
\hline No.opt & & & & & 5 & - & 4 & - & 5 & - & 5 & - & 5 & - \\
\hline
\end{tabular}

In (15), $\sum_{(i, j) \in E_{R} \cup A_{R}} d(i, j)$ is the overall demand of an instance. Correspondingly, $\left\lceil\sum_{(i, j) \in E_{R} \cup A_{R}} d(i, j) / Q\right\rceil$ is the minimal number of routes required for the instance, because the load of a single route must not exceed $Q$. Hence, the second term of (15) is the average load of each route, and thereby, $A R$ is the average residual. A task is defined as a free task if its 
TABLE VII

RESUlts FOR BEUllens et al. SET F. "mean", "No.opt", AND "APD” STAND FOR AVERAGe Cost ANd Runtimes, Number of OPTIMAL SOLUTIONS, AND APD to THE LOWER BOUNDS, RESPECTIVELY

\begin{tabular}{|c|c|c|c|c|c|c|c|c|c|c|c|c|c|c|}
\hline \multirow{2}{*}{ Name } & \multirow[t]{2}{*}{$|V|$} & \multirow[t]{2}{*}{$|R|$} & \multirow[t]{2}{*}{$|\mathrm{E}|$} & \multirow[t]{2}{*}{ LB } & \multicolumn{2}{|r|}{ GLS } & \multicolumn{2}{|c|}{ TSA1 } & \multicolumn{2}{|c|}{ TSA 2} & \multicolumn{2}{|r|}{ RTS } & \multicolumn{2}{|c|}{ RTS* $^{*}$} \\
\hline & & & & & Cost & $\mathrm{CPU}(\mathrm{s})$ & Cost & $\mathrm{CPU}(\mathrm{s})$ & Cost & $\mathrm{CPU}(\mathrm{s})$ & Cost & $\mathrm{CPU}(\mathrm{s})$ & Cost & $\mathrm{CPU}(\mathrm{s})$ \\
\hline F1 & 73 & 85 & 105 & 1065 & 1065 & 0.9 & 1090 & 8.1 & 1085 & 62.0 & 1155 & 3.3 & 1065 & 30.8 \\
\hline F2 & 58 & 58 & 81 & 920 & 920 & 0.6 & 940 & 1.8 & 920 & 0.2 & 920 & 0.8 & 920 & 0.8 \\
\hline F3 & 46 & 47 & 61 & 400 & 400 & 0.0 & 480 & 1.3 & 400 & 0.9 & 400 & 0.4 & 400 & 0.4 \\
\hline F4 & 70 & 77 & 99 & 930 & 940 & 62.0 & 970 & 1.9 & 960 & 44.6 & 950 & 5.8 & 950 & 28.9 \\
\hline F5 & 68 & 61 & 94 & 1180 & 1180 & 0.1 & 1185 & 1.3 & 1180 & 12.0 & 1180 & 0.5 & 1180 & 0.5 \\
\hline $\mathrm{F} 6$ & 49 & 43 & 66 & 490 & 490 & 0.0 & 540 & 0.3 & 490 & 0.0 & 490 & 0.4 & 490 & 0.4 \\
\hline F7 & 73 & 50 & 94 & 1080 & 1080 & 0.0 & 1110 & 2.5 & 1080 & 1.2 & 1080 & 0.0 & 1080 & 0.0 \\
\hline F8 & 74 & 59 & 98 & 1135 & 1145 & 46.4 & 1155 & 0.8 & 1145 & 25.0 & 1145 & 1.0 & 1145 & 11.6 \\
\hline F9 & 93 & 103 & 141 & 1145 & 1145 & 0.9 & 1520 & 5.2 & 1170 & 102.1 & 1215 & 11.3 & 1145 & 29.6 \\
\hline F10 & 56 & 49 & 76 & 1010 & 1010 & 0.0 & 1010 & 0.1 & 1010 & 0.1 & 1010 & 0.0 & 1010 & 0.0 \\
\hline F11 & 80 & 94 & 113 & 1015 & 1015 & 1.1 & 1100 & 6.0 & 1015 & 88.1 & 1015 & 7.6 & 1015 & 7.6 \\
\hline F12 & 74 & 67 & 103 & 900 & 910 & 57.2 & 1000 & 3.4 & 910 & 33.5 & 910 & 3.0 & 910 & 22.5 \\
\hline F13 & 49 & 52 & 73 & 835 & 835 & 0.1 & 855 & 0.6 & 835 & 0.1 & 835 & 0.1 & 835 & 0.1 \\
\hline F14 & 53 & 55 & 72 & 1025 & 1025 & 4.7 & 1085 & 1.8 & 1035 & 20.8 & 1125 & 2.0 & 1035 & 12.8 \\
\hline F15 & 85 & 107 & 126 & 945 & 945 & 0.3 & 1315 & 15.5 & 990 & 101.8 & 975 & 6.6 & 945 & 69.3 \\
\hline F16 & 60 & 54 & 80 & 775 & 775 & 0.0 & 945 & 1.3 & 775 & 2.4 & 775 & 0.4 & 775 & 0.4 \\
\hline F17 & 38 & 36 & 50 & 605 & 605 & 0.0 & 660 & 0.2 & 630 & 3.3 & 605 & 0.1 & 605 & 0.1 \\
\hline F18 & 78 & 88 & 110 & 835 & 850 & 68.6 & 945 & 9.7 & 850 & 65.0 & 850 & 4.6 & 850 & 38.0 \\
\hline F19 & 77 & 66 & 103 & 685 & 725 & 39.5 & 740 & 1.1 & 740 & 24.4 & 725 & 2.0 & 725 & 19.3 \\
\hline F20 & 56 & 63 & 80 & 610 & 610 & 0.3 & 610 & 0.2 & 610 & 6.9 & 610 & 0.6 & 610 & 0.6 \\
\hline F21 & 57 & 72 & 82 & 905 & 905 & 1.0 & 940 & 1.5 & 905 & 31.9 & 905 & 1.0 & 905 & 1.0 \\
\hline F22 & 54 & 44 & 73 & 790 & 790 & 0.1 & 790 & 0.1 & 790 & 0.3 & 790 & 0.1 & 790 & 0.1 \\
\hline F23 & 93 & 89 & 130 & 705 & 725 & 79.9 & 895 & 3.2 & 730 & 56.1 & 775 & 7.8 & 725 & 41.8 \\
\hline F24 & 97 & 86 & 142 & 975 & 975 & 6.7 & 1040 & 7.2 & 1010 & 55.4 & 1005 & 7.4 & 1005 & 37.6 \\
\hline F25 & 26 & 28 & 35 & 430 & 430 & 0.0 & 430 & 0.0 & 430 & 0.1 & 430 & 0.0 & 430 & 0.0 \\
\hline mean & & & & 855.6 & 859.8 & 14.8 & 934.0 & 3.0 & 867.8 & 29.5 & 875 & 2.7 & 861.8 & 14.2 \\
\hline No.opt & & & & & 19 & - & 4 & - & 13 & - & 14 & - & 16 & - \\
\hline $\mathrm{APD}$ & & & & - & 0.54 & . & 8.71 & . & 1.44 & 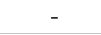 & 2.09 & 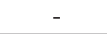 & 0.75 & - \\
\hline
\end{tabular}

TABLE VIII

REsults For Brandão And Eglese’s Benchmark Test Set. “mean” Stands for Average Cost and Runtimes

\begin{tabular}{|c|c|c|c|c|c|c|c|c|c|}
\hline \multirow[t]{2}{*}{ Name } & \multirow[t]{2}{*}{$|V|$} & \multirow[t]{2}{*}{$|R|$} & \multirow[t]{2}{*}{$|E|$} & \multicolumn{2}{|c|}{ TSA1 } & \multicolumn{2}{|c|}{ RTS } & \multicolumn{2}{|c|}{ RTS* $^{*}$} \\
\hline & & & & Cost & $\mathrm{CPU}(\mathrm{s})$ & Cost & $\mathrm{CPU}(\mathrm{s})$ & Cost & $\mathrm{CPU}(\mathrm{s})$ \\
\hline G1-A & 255 & 347 & 375 & 1049708 & 552.6 & 1042411 & 348.1 & 1025765 & 1778.4 \\
\hline G1-B & 255 & 347 & 375 & 1140692 & 607.1 & 1137402 & 409.3 & 1135873 & 1905.2 \\
\hline $\mathrm{G} 1-\mathrm{C}$ & 255 & 347 & 375 & 1282270 & 643.4 & 1278700 & 401.7 & 1271894 & 1903.5 \\
\hline G1-D & 255 & 347 & 375 & 1420126 & 595.6 & 1417963 & 444.8 & 1402433 & 2230.6 \\
\hline $\mathrm{G} 1-\mathrm{E}$ & 255 & 347 & 375 & 1583133 & 470.5 & 1573141 & 534.1 & 1558548 & 2279.9 \\
\hline $\mathrm{G} 2-\mathrm{A}$ & 255 & 375 & 375 & 1129229 & 1018.9 & 1126112 & 510.7 & 1125602 & 2225.5 \\
\hline $\mathrm{G} 2-\mathrm{B}$ & 255 & 375 & 375 & 1255907 & 785.6 & 1245141 & 454.4 & 1242542 & 2242.6 \\
\hline $\mathrm{G} 2-\mathrm{C}$ & 255 & 375 & 375 & 1418145 & 594.3 & 1416282 & 473.0 & 1401583 & 2530.4 \\
\hline G2-D & 255 & 375 & 375 & 1516103 & 1263.7 & 1516072 & 524.1 & 1516072 & 2336.1 \\
\hline $\mathrm{G} 2-\mathrm{E}$ & 255 & 375 & 375 & 1701681 & 615.9 & 1684472 & 525.6 & 1668348 & 2492.1 \\
\hline mean & & & & 1349699.1 & 714.8 & 1343769.6 & 462.6 & 1334866.0 & 2192.4 \\
\hline
\end{tabular}

TABLE IX

AVERAGE RUNTIMES OF RTS* AND GRO (IN CPU SECONDS)

\begin{tabular}{cccc}
\hline Name & $\begin{array}{c}\text { Average Runtime } \\
\text { of RTS* }\end{array}$ & $\begin{array}{c}\text { Average Runtime } \\
\text { of GRO }\end{array}$ & $\begin{array}{c}\text { Prop of } \\
\text { GRO }\end{array}$ \\
\hline gdb & 0.6188 & 0.0095 & $1.5317 \%$ \\
val & 6.8253 & 0.0092 & $0.1349 \%$ \\
egl & 125.7910 & 2.9417 & $2.3386 \%$ \\
Beullens et al.'s Set C & 27.1307 & 0.6334 & $2.3348 \%$ \\
Beullens et al.'s Set D & 17.3051 & 0.0295 & $0.1704 \%$ \\
Beullens et al.'s Set E & 26.0732 & 0.2419 & $0.9278 \%$ \\
Beullens et al.'s Set F & 14.1632 & 0.0155 & $0.1093 \%$ \\
Brandão\&Eglese & 462.5752 & 18.1386 & $3.9212 \%$ \\
Overall & & & \\
& 52.3970 & 1.4425 & $2.7531 \%$
\end{tabular}

demand is no more than the $A R$. Based on such definition, the If the constraints are so tight that the solutions obtained by numerator of PFT stands for the number of free tasks in an GRO are not even close to the feasible region (i.e., the violainstance. A smaller PFT implies a tighter capacity constraint.

Intuitively speaking, the tighter the capacity constraints, the harder the GRO can successfully repair an infeasible solution. tions are still large after the repair process), GRO is unlikely to benefit the search process much. Hence, we expect GRO to be more preferable for problem instances whose capacity 
constraints are not too tight, i.e., with large PFTs. In order to verify this hypothesis, we calculated the correlation coefficient between the solution quality and PFT based on the total 191 problem instances involved in this paper. First, the costs of the solutions are divided by the lower bounds for normalization. The correlation coefficient between the normalized results and PFT is -0.2463 for RTS and -0.2306 for RTS* . This clearly implies negative correlation between PFT and the performance of RTS, and thereby, our hypothesis is validated.

\section{CONCLUSION AND Discussion}

In this paper, we proposed a search operator called GRO for CARP based on the vertex encoding. GRO is motivated by the observation that, in the vertex encoding, an ordered list may correspond to many solutions with different zero-one variables, and the traditional move operators may not be able to focus on the low cost ordered lists and lead to an ineffective search. GRO tries to minimize the capacity violation of a low cost infeasible solution while retaining its ordered list, which is formulated as a bin-packing problem and is NP-hard [30]. GRO employs an insertion heuristic and a tabu search to find near-optimal solutions for the bin-packing problem. To verify the efficacy of GRO, we embedded it in TSA1 and tested the resultant RTS algorithm on five previously studied benchmark test sets, which contain 191 CARP instances in total. Experimental results showed that GRO enhanced TSA1 significantly both in terms of solution quality and computational time, and the RTS* algorithm provided very competitive results in comparison to a number of state-of-the-art approaches for CARP. Furthermore, GRO is independent of the method generating the infeasible solutions and can be easily embedded in any method utilizing the vertex encoding (e.g., the most straightforward way is to call the GRO whenever an infeasible solution is reached.). Therefore, we believe that GRO would be an effective supplementary operator to the existing algorithms.

Two parameters need to be predefined for GRO: the tabu tenure and the number of iterations. In this paper, they are arbitrarily set to $F / 2$ and $N$. Although these values are not meant to be optimal, experimental study showed that GRO is not very sensitive to the parameters. Hence, $F / 2$ and $N$ can be used as a rule of thumb.

As mentioned in Section II-B, few repair operators have been specifically designed for CARP. However, in the broader context of heuristic search, lots of repair operators have been proposed for various optimization problems [31]-[38]. Moreover, it is usually the case that a repair operator for one combinatorial optimization problem may not be directly applied to another problem, and some modifications are required. Hence, at the first sight, GRO merely looks like a new repair operator whose usage is restricted within a specific domain. However, the underlying mechanism of GRO is essentially different from many existing repair operators. When applied to an infeasible solution, GRO does not search in the original solution space (i.e., the space represented by the vertex encoding) for the alternative feasible solution. Instead, the search is carried out in the space represented by the zero-one variables. That means that GRO first switches the search space, then conducts the search, and finally transforms the obtained solution back to the original solution space. In consequence, after the repair process, we are likely to obtain a solution that is "distant" from the original solution in the solution space. Therefore, unlike many existing repair operators for combinatorial optimization (e.g., [34], [35], and [37]), which mainly conduct local searches in the original solution space, GRO actually implements a relatively global search. Such a characteristic, as well as the promising performance exhibited by GRO, may hopefully shed some light on the design of repair operators for other combinatorial optimization problems.

Last but not least, the promising results obtained via GRO imply that the assignment of tasks in different routes (i.e., zero-one variables) might have been overlooked in previous studies. Hence, the following question arises: Can an encoding scheme which focuses more on the assignment of tasks be more effective than the vertex encoding? This issue deserves in-depth research in the future.

\section{ACKNOWLEDGMENT}

The authors would like to thank Dr. J. Brandão and Prof. R. Eglese for the kind sharing of the test sets used in their work and the anonymous reviewers for the helpful comments and criticisms.

\section{REFERENCES}

[1] M. Dror, Ed., Arc Routing: Theory, Solutions and Applications. Boston, MA: Kluwer, 2000

[2] B. L. Golden and R. T. Wong, "Capacitated arc routing problems," Networks, vol. 11, no. 3, pp. 305-315, 1981

[3] B. L. Golden, J. S. DeArmon, and E. K. Baker, "Computational experiments with algorithms for a class of routing problems," Comput. Oper. Res., vol. 10, no. 1, pp. 47-59, 1983.

[4] G. Ulusoy, "The fleet size and mix problem for capacitated arc routing," Eur. J. Oper. Res., vol. 22, no. 3, pp. 329-337, Dec. 1985.

[5] W. L. Pearn, "Approximate solutions for the capacitated arc routing problem," Comput. Oper. Res., vol. 16, no. 6, pp. 589-600, 1989.

[6] W. L. Pearn, "Augment-insert algorithms for the capacitated arc routing problem," Comput. Oper. Res., vol. 18, no. 2, pp. 189-198, Feb. 1991.

[7] M. C. Mourao and L. Amado, "Heuristic method for a mixed capacitated arc routing problem: A refuse collection application," Eur. J. Oper. Res., vol. 160, no. 1, pp. 139-153, Jan. 2005.

[8] S. K. Amponsah and S. Salhi, "The investigation of a class of capacitated arc routing problems: The collection of garbage in developing countries,' Waste Manage., vol. 24, no. 7, pp. 711-721, 2004.

[9] A. Hertz, G. Laporte, and M. Mittaz, "A tabu search heuristic for the capacitated arc routing problem," Oper. Res., vol. 48, no. 1, pp. 129-135, Jan./Feb. 2000.

[10] A. Hertz and M. Mittaz, "A variable neighborhood descent algorithm for the undirected capacitated arc routing problem," Transp. Sci., vol. 35, no. 4, pp. 425-434, Nov. 2001.

[11] P. Greistorfer, "A tabu scatter search metaheuristic for the arc routing problem," Comput. Ind. Eng., vol. 44, no. 2, pp. 249-266, Feb. 2003.

[12] P. Lacomme, C. Prins, and W. Ramdane-Cherif, "Competitive memetic algorithms for arc routing problem," Ann. Oper. Res., vol. 131, no. 1-4, pp. 159-185, Oct. 2004.

[13] P. Lacomme, C. Prins, and W. Ramdane-Cherif, "A genetic algorithm for the capacitated arc routing problem and its extensions," in Proc. EvoWorkshops Appl. Evol. Comput., Como, Italy, 2001, pp. 473-483.

[14] P. Beullens, L. Muyldermans, D. Cattrysse, and D. V. Oudheusden, "A guided local search heuristic for the capacitated arc routing problem," Eur. J. Oper. Res., vol. 147, no. 3, pp. 629-643, Jun. 2003.

[15] H. Handa, D. Lin, L. Chapman, and X. Yao, "Robust solution of salting route optimization using evolutionary algorithms," in Proc. IEEE Congr. Evol. Comput., Vancouver, BC, Canada, 2006, pp. 3098-3105. 
[16] H. Handa, L. Chapman, and X. Yao, "Robust route optimization for gritting/salting trucks: A CERCIA experience," IEEE Comput. Intell. Mag., vol. 1, no. 1, pp. 6-9, Feb. 2006.

[17] J. Brandão and R. Eglese, "A deterministic tabu search algorithm for the capacitated arc routing problem," Comput. Oper. Res., vol. 35, no. 4, pp. 1112-1126, Apr. 2008.

[18] T. Z. Jiang and F. G. Yang, "An evolutionary tabu search for cell image segmentation," IEEE Trans. Syst., Man, Cybern. B, Cybern., vol. 32, no. 5, pp. 675-678, Oct. 2002.

[19] S. Pierre and F. Houeto, "Assigning cells to switches in cellular mobile networks using taboo search," IEEE Trans. Syst., Man, Cybern. B, Cybern., vol. 32, no. 3, pp. 351-356, Jun. 2002.

[20] G. N. Frederickson, "Approximation algorithms for some postman problems," J. ACM, vol. 26, no. 3, pp. 538-554, Jul. 1979.

[21] J. S. DeArmon, "A comparison of heuristics for the capacitated Chinese postman problems," M.S. thesis, Univ. Maryland, College Park, MD, 1981.

[22] E. Benavent, V. Campos, E. Corberán, and E. Mota, "The capacitated arc routing problem: Lower bounds," Networks, vol. 22, no. 7, pp. 669-690, Dec. 1992

[23] R. W. Eglese, "Routing winter gritting vehicles," Discrete Appl. Math., vol. 48, no. 3, pp. 231-244, Feb. 1994.

[24] R. W. Eglese and L. Y. O. Li, "A tabu search based heuristic for arc routing with a capacity constraint and time deadline," in Metaheuristics: Theory and Applications, I. H. Osman and J. P. Kelly, Eds. Boston, MA: Kluwer, 1996, pp. 633-650.

[25] L. Y. O. Li and R. W. Eglese, "An interactive algorithm for vehicle routing for winter-gritting," J. Oper. Res. Soc., vol. 47, no. 2, pp. 217-228, 1996.

[26] J. M. Belenguer and E. Benavent, "A cutting plane algorithm for the capacitated arc routing problem," Comput. Oper. Res., vol. 30, no. 5, pp. 705-728, Apr. 2003.

[27] D. Ahr, "Contributions to multiple postmen problems," Ph.D. dissertation, Rupercht-Karls-Universitat, Heidelberg, Germany, 2004.

[28] R. Baldacci and V. Maniezzo, "Exact methods based on node-routing formulations for undirected arc-routing problems," Networks, vol. 47, no. 1, pp. 52-60, 2006.

[29] H. Longo, D. A. M. Poggi, and E. Uchoa, "Solving capacitated arc routing problems using a transformation to the CVRP," Comput. Oper. Res., vol. 33, no. 6, pp. 1823-1837, Jun. 2006.

[30] E. G. Coffman, M. R. Garey, and D. S. Johnson, "Approximation algorithms for bin-packing-An updated survey," in Algorithm Design for Computer System Design, G. Ausiello, M. Lucertini, and P. Serafini, Eds. Berlin, Germany: Springer-Verlag, 1984, pp. 49-106.

[31] Z. Michalewicz and G. Nazhiyath, "Genocop III: A co-evolutionary algorithm for numerical optimization problems with nonlinear constraints," in Proc. IEEE Int. Conf. Evol. Comput., Perth, Australia, 1995, vol. 2, pp. 647-651.

[32] K. Harada, J. Sakuma, I. Ono, and S. Kobayashi, "Constraint-handling method for multi-objective function optimization: Pareto descent repair operator," in Proc. 4th Int. Conf. Evol. Multi-Criterion Optimization, Sendai, Japan, 2007, pp. 156-170.

[33] H. Handa, K. Watanabe, O. Katai, T. Konishi, and M. Baba, "Coevolutionary genetic algorithm for constraint satisfaction with a genetic repair operator for effective schemata formation," in Proc. IEEE Int. Conf. Syst., Man, Cybern., Tokyo, Japan, 1999, vol. 3, pp. 616-621.

[34] P. C. Chu and J. E. Beasley, "A genetic algorithm for the multidimensional knapsack problem," J. Heuristics, vol. 4, no. 1, pp. 63-86, Jun. 1998.

[35] M. Gröbner and P. Wilke, "Optimizing employee schedules by a hybrid genetic algorithm," in Proc. EvoWorkshops Appl. Evol. Comput., 2001, vol. 2037, pp. 463-472.

[36] Q. Zhang, J. Sun, and E. Tsang, "An evolutionary algorithm with guided mutation for the maximum clique problem," IEEE Trans. Evol. Comput., vol. 9, no. 2, pp. 192-200, Apr. 2005.

[37] H. Ueda, D. Ouchi, K. Takahashi, and T. Miyahara, "A co-evolving timeslot/room assignment genetic algorithm technique for university timetabling," in Proc. Practice Theory Automated Timetabling III, 2001, vol. 2079, pp. 48-63.

[38] C. A. Coello Coello, "Theoretical and numerical constraint-handling techniques used with evolutionary algorithms: A survey of the state of the art," Comput. Methods Appl. Mech. Eng., vol. 191, no. 11/12, pp. 1245-1287, Jan. 2002.

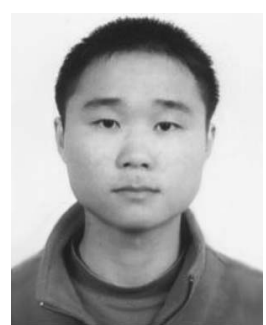

Yi Mei received the B.S. degree in mathematics from the University of Science and Technology of China, Hefei, China, in 2005, where he is currently working toward the Ph.D. degree in the Nature Inspired Computation and Applications Laboratory, Department of Computer Science and Technology.

His current research interests include memetic algorithm, tabu search, and other metaheuristics for solving arc routing problem.

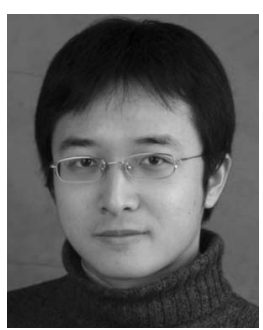

Ke Tang (M'07) received the B.Eng. degree from the Department of Control Science and Engineering, Huazhong University of Science and Technology, Wuhan, China, in 2002 and the Ph.D. degree from the School of Electrical and Electronic Engineering, Nanyang Technological University, Singapore, in 2007.

$\mathrm{He}$ is currently an Associate Professor with the Department of Computer Science and Technology, University of Science and Technology of China, Hefei, China. His major research interests include machine learning, pattern analysis, evolutionary computation, data mining, metaheuristic algorithms, and real-world applications.

Dr. Tang is a member of the Computational Intelligence Society of IEEE and the Chair of IEEE Task Force on Large Scale Global Optimization.

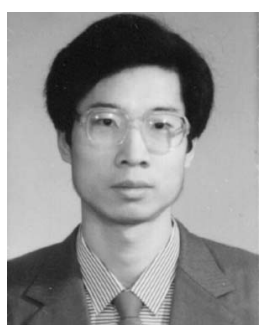

Xin Yao (M'91-SM'96-F'03) received the B.Sc. degree from the University of Science and Technology of China (USTC), Hefei, China, in 1982, the M.Sc. degree from the North China Institute of Computing Technology, Beijing, China, in 1985, and the Ph.D. degree from USTC in 1990.

From 1985 to 1990 , he was an Associate Lecturer and Lecturer with USTC, while working toward the Ph.D. degree on simulated annealing and evolutionary algorithms. He took up a Postdoctoral Fellowship in the Computer Sciences Laboratory, Australian National University, Canberra, A.C.T., Australia, in 1990, and continued his work on simulated annealing and evolutionary algorithms. $\mathrm{He}$ was with the Knowledge-Based Systems Group, Commonwealth Scientific and Industrial Research Organization Division of Building, Construction and Engineering, Melbourne, Vic., Australia, in 1991, working primarily on an industrial project on automatic inspection of sewage pipes. He returned to Canberra in 1992 to take up a lectureship in the School of Computer Science, University College [University of New South Wales at Australian Defense Force Academy (ADFA)], ADFA, Canberra, where he was later promoted to a Senior Lecturer and Associate Professor. Attracted by the English weather, he moved to the University of Birmingham, Birmingham, U.K., as a Professor of computer science in 1999. He is currently the Director of the Centre of Excellence for Research in Computational Intelligence and Applications, School of Computer Science, University of Birmingham, and a Changjiang (Visiting) Chair Professor (Cheung Kong Scholar) at USTC. He is an Associate Editor or Editorial Board Member of ten journals and the Editor of the World Scientific Book Series on Advances in Natural Computation. He has given more than 50 invited keynote and plenary speeches at conferences and workshops worldwide. His major research interests include evolutionary artificial neural networks, automatic modularization of machine learning systems, evolutionary optimization, constraint handling techniques, computational time complexity of evolutionary algorithms, coevolution, iterated prisoner's dilemma, data mining, and real-world applications. He has more than 250 refereed publications.

Dr. Yao was awarded the President's Award for Outstanding Thesis by the Chinese Academy of Sciences for his Ph.D. work on simulated annealing and evolutionary algorithms in 1989. He won the 2001 IEEE Donald G. Fink Prize Paper Award for his work on evolutionary artificial neural networks. $\mathrm{He}$ is the Editor-in-Chief of the IEEE TRANSACTIONS ON EVOLUTIONARY COMPUTATION. 